\title{
TIEMPO Y DISCIPLINA EN GERENTES DE EMPRESAS TRANSNACIONALES EN BUENOS AIRES
}

\section{TIME AND DISCIPLINE AMONG MANAGERS OF TRANSNATIONAL ENTERPRISES IN BUENOS AIRES}

\section{Diego Szlechter*}

\begin{abstract}
RESUMEN
El presente trabajo analiza el modo de utilización y de concepción del tiempo de altos directivos de empresas transnacionales en la Argentina. Nos basaremos, por un lado, en un debate teórico en torno a la noción de tiempo desde diferentes disciplinas (antropología, sociología, historia de la vida cotidiana), cotejándolo con fuentes primarias y secundarias de las mismas empresas. El objetivo de este estudio radica en encontrar tendencias generales en la noción y utilización del tiempo en altos directivos y mandos medios dentro del capitalismo actual. Las prácticas cotidianas de estos quedan, en gran medida, subordinados o subsumidos a los intereses corporativos. De esta manera, el eje alrededor del cual gira la temporalidad constituye la idea de la urgencia (de agregar valor al tiempo).
\end{abstract}

PALABRAS CLAVE: ARGENTINA * EMPRESAS TRANSNACIONALES * CAPITALISMO * TRABAJO $*$ GERENTES $*$ MANDOS MEDIOS $*$ ADMINISTRACIÓN DEL TIEMPO

\section{ABSTRACT}

This work analyzes the way managers of transnational companies in Argentina use and conceive their time. We will focus, on one hand, on the theoretic debate of the notion of time from different perspectives (anthropology, sociology and history of everyday life), comparing it with primary and secondary sources of those companies. The objective of this research relies upon finding general tendencies in the notion and use of time among high level executives and middle line managers in the current capitalism. The everyday practices of this people remain, in some way, subordinated or subsumed to the corporate interests. Therefore, the idea of temporality is shaped by the notion of urgency (of adding value to time).

KEYWORDS: ARGENTINA $*$ TRANSNATIONAL CORPORATIONS $*$ CAPITALISM $*$ LABOUR $*$ MANAGERS $*$ MIDDLE MANAGEMENT $*$ TIME MANAGEMENT 


\section{INTRODUCCIÓN Y PRESENTACIÓN DE LA PROBLEMÁTICA}

El presente trabajo pretende analizar los modos a través de los cuales los cuadros ${ }^{1}$ de empresas transnacionales en Buenos Aires internalizan y naturalizan cierta disciplina respecto a tiempos de trabajo y del tiempo libre, de forma que les impiden su clara diferenciación. El camino que seguiremos en la siguiente exposición será caracterizar a la figura del manager o mando medio, ampliamente trabajada desde la sociología por Bouffartigue et ál. (2001), Bouffartigue (2001), Brunel (2004), Gadéa (2003), Livian et ál. (2006) y desde la antropología por Flament (2002), posteriormente presentar un debate teórico en torno a la noción de tiempo (de trabajo y de no trabajo) en el capitalismo actual y por último, indagar cuáles son algunas de las formas en que se manifiestan rasgos de introyección de una disciplina temporal en los cuadros de empresas transnacionales. Por último, presentaremos algunas interrogantes que se planteen a lo largo de la discusión teórica que puedan llevar a una investigación de tipo empírica que logre responder de forma más acabada a dichas cuestiones. En esta instancia, sólo dejaré formuladas algunas preguntas que pueden allanar el camino para profundizar en estos temas.

Si bien la elección de los sujetos de estudio - los mandos medios de las empresas

Es necesario aclarar a qué nos referimos con cuadros: Boltanski y Chiapello (2002) sostienen que el término cuadro, a partir de la década de los 90 , pasa a constituir un término obsoleto, debido a que sugiere jerarquía y burocracia, términos rechazados por el discurso del management debido a que es la autonomía y la horizontalidad lo que prima en el discurso actual. Incluso, según sostienen los autores, la literatura de los 90 reemplazan a los cuadros por el personal intermedio $y$ subalterno. Por lo tanto, al hablar de cuadro, estamos haciendo referencia a lo que en la jerga empresarial suele llamarse manager en su acepción más general, lo cual incluye a aquellos que tienen la capacidad de animar equipos, manejar personas, etc. Como sostienen los autores, el manager, en el capitalismo actual, es el hombre de las redes que en lugar de hacer carrera, construye relaciones en base a sus competencias y carisma. transnacionales - se debe a la intención de comprender tendencias de cambio globales, la delimitación geográfica a la ciudad de Buenos Aires, aunque resuma en sí misma el conjunto de transformaciones globales del capitalismo, evita la tendencia que poseen los enfoques globales, los cuales suelen presentar a los factores explicativos del cambio como "fuerzas ajenas a los seres humanos y a las naciones, que se verían de esta forma obligadas a padecerlos del mismo modo que se soporta una tormenta" (Boltanski y Chiapello, 2002: 34). La percepción de inevitabilidad ${ }^{2}$ puede terminar llevando a la justificación.

La razón por la cual el trabajo empírico que se pretende llevar a cabo se centra en empresas transnacionales (las cuales representan a su vez, la mayor parte del comercio mundial en su totalidad), se debe a que las justificaciones que encuentran sus actores muestran competencias que son constantemente reproducidas a través de los procesos de socialización familiares y escolares (Boltanski y Chiapello, 2002: 47). Tal como sostiene López Ruiz (2004), la mutación sufrida por el espíritu capitalista a través de la reformulación neoliberal de la economía política, generó una explicación de la realidad que toma a la figura del ejecutivo como una matriz de conducta a ser diseminada por la sociedad entera, la cual no se funda en la satisfacción del deseo, sino más bien en la inversión constante y exclusiva de la voluntad en la producción de riqueza abstracta. La ganancia se torna no una obligación sino un imperativo vital, la lógica del capital pasa a ser la razón de su existencia.

Este compromiso asumido por estos "empleados capitalistas" debe lograrse por medios pacíficos y no por coacción. Durand (2006) llama a este compromiso "servilismo

2 Bessin (1998) considera que lo que diferencia al posfordismo del taylorismo o fordismo es la dependencia del entorno. A la aceleración de las operaciones de producción tayloristas se suma otra forma de aceleración producto de los cambios en el entorno, que a su vez obliga a las empresas a modificar la esquematización del tiempo. De la organización secuencial, se pasa a la organización de la simultaneidad de operaciones. Este nuevo tipo de aceleración en las empresas, aumenta la disposición a la irreversibilidad. 
voluntario". Debe lograrse una adhesión plena y conciente a los objetivos de la empresa, que requiere de sacrificios (idealmente, consentidos libremente) $y$ de iniciativas que lleven a la estabilización del sistema en pos de su producción $y$ reproducción. Estos empleados deben encontrar maneras de justificar ideológicamente esta adhesión: "las personas necesitan poderosas razones morales para adherirse al capitalismo" (Boltanski y Chiapello, 2002: 43).

En orden de mantener vivo su espíritu $y$ poder de movilización ${ }^{3}$, el capitalismo debe apropiarse de recursos discursivos ajenos al mismo e incluso que le son hostiles. Así surgen, por ejemplo, los programas de responsabilidad social empresarial, las fundaciones empresarias caritativas y filantrópicas o las mismas declaraciones de principios de las empresas (llamadas por Estas "misión" o "visión"). Para atraer a las elites al mundo de los negocios y hacer de ellos sujetos productivos, es necesario que las empresas les ofrezcan los aportes que esta le hace a la sociedad en su conjunto $y$ hacerles creer que el objetivo de los negocios no es sólo ganar dinero (Boltanski y Chiapello, 2002: 106). De Gaulejac (2005) define a esta movilización como "ideología de la gestión". Es necesario aclarar que el capitalismo no está regido por normas de justicia (no es intrínsecamente injusto, sino que la justicia no es pertinente dentro de sus reglas de juego $)^{4}$, debido a que la norma de acumulación

$3 \quad$ El poder de movilización que intenta producir el capitalismo está dirigido en un doble sentido: por un lado, se hace necesario movilizar a los cuadros de las empresas, pero por otro, se hace no menos perentorio aplicar dispositivos de control desmovilizadores para contrarrestar los intentos de movilización de la clase trabajadora, a veces con éxito y otras veces no, pero el objetivo se encamina, en todos los niveles jerárquicos, a una individualización de la relación del trabajador (o de los cuadros) para con "su" empresa.

Tal como veremos al tratar el tema de la temporalidad en el capitalismo actual, esta falta de necesidad de imaginar un orden justo (o más justo), se relaciona con un determinado modelo temporal, en el cual se profundiza la brecha entre la experiencia acumulada y el futuro incierto, sobredimensionándose el tiempo presente. De esta manera, se llega a naturalizar el orden social dominante $y$ poco se hace por imaginarse un orden social deseado. de capital es amoral, lo que obliga al capitalismo (a las empresas $y$ a sus cuadros) a justificarse $y$ autocontrolarse (Boltanski y Chiapello, 2002: 82 y 83).

Por otro lado, López Ruiz, en lugar de hablar de las justificaciones que debe encontrar el capitalismo para lograr su objetivo, sostiene que este obliga al trabajador a adoptar determinadas estrategias de superviviencia dentro de un ambiente que constantemente lo pone a prueba (en términos de los resultados de sus inversiones). El trabajador debe convertirse en un trabajador-empresario. El autor caracteriza al capitalismo moderno como la masificación del afán de lucro en todos los niveles jerárquicos. Cada uno debe internalizar el afán de lucro $y$ hacerlo su principal motivo, tal que cada uno se convierta en empresario de sí mismo.

Para la empresa transnacional, dentro de un marco de economía neoliberal, el salario de sus managers es una renta, el producto o el rendimiento de un capital. Si esta "máquina" llamada trabajador, debe producir flujos de renta, entonces este debe convertirse en una especie de empresa: la base de la sociedad ya no es el individuo, sino el trabajador-empresa (López Ruiz, 2004).

\section{EL TIEMPO EN LA EMPRESA}

El tiempo es pensado y experimentado de diversas maneras de acuerdo con el grupo social al cual un individuo pertenece. Esta diversidad no sólo proviene de criterios internos de la estructura simbólica de los grupos sociales, sino que además es producto de las relaciones jerárquicas de una sociedad basada en las clases.

La utilización del tiempo es expresiva de la forma que toman los diferentes tipos de relaciones sociales. Así, las definiciones sociales de tiempo o temporalidades son fuertemente marcadas por la experiencia de clase social, género y grupo etario (Heilborn, citado por Delfino, 2004).

La percepción del tiempo está condicionada por el lugar que ocupa la noción de trabajo 
en la vida de las personas y por las expectativas depositadas en torno a este. De esta forma, se jerarquizan las actividades de la vida cotidiana.

Thompson (1967) sostenía que, en las sociedades campesinas ${ }^{5}$, la percepción del tiempo se relacionaba con la orientación a las tareas. $\mathrm{El}$ autor propone tres explicaciones a esta forma de comportamiento: 1) es más comprensible, en términos humanos, que el trabajo pautado, ya que el campesino parece atender lo que son necesidades observadas y explícitas; 2) dentro de una comunidad en la cual la orientación a la tarea es común, parece haber una menor demarcación entre "trabajo" y "vida". Las relaciones sociales $y$ el trabajo se entrecruzan (el día de trabajo se prolonga o acorta de acuerdo con la tarea realizada), por lo que no existe conflicto entre trabajo y "pasar del día" y 3) para los hombres acostumbrados al trabajo pautado por el reloj, esta actitud hacia el trabajo aparece como derrochadora $y$ falto de urgencia (Thompson, 1967: 60).

Si el capitalismo actual muestra una característica propia de las sociedades campesinas - la turbia escisión entre trabajo y tiempo libre - no se debe a una vuelta al pasado, sino precisamente a una evolución en el proceso de disciplinamiento del tiempo: si en el capitalismo en red (Boltanski y Chiapello, 2002), trabajo y "vida" se entrecruzan, se debe a que los cuadros de las empresas internalizan una cierta noción de tiempo que abarca el tiempo laboral y el de fuera del trabajo: las redes se construyen en cualquier momento $y$ en cualquier lugar. Toda acción, va a estar sometida a las leyes del mercado, incluso la misma división entre tiempo de trabajo y tiempo libre. El tiempo libre y el de trabajo tienen una conexión y un orden, que impone el trabajo, siendo este último el que controla

En general, al referirse a las sociedades pre-industriales, Thompson sostiene que el patrón que describía la forma de trabajo de estas, era la alternancia de momentos de trabajo intenso y de ocio $y$ en la cual los hombres estaban en control de sus propias vidas laborales. Como este patrón de trabajo subsiste entre algunos artistas, escritores, pequeños agricultores e incluso estudiantes, el autor eleva la provocadora pregunta acerca de si en realidad esto sería un ritmo de trabajo humano "natural" (Thompson, 1967: 73). y establece la forma en que el tiempo libre deba ser utilizado de la mejor manera para reproducir la vida y servir a que los trabajadores rindan más en el ámbito de trabajo (Sgrazzutti, 2004). "Es preciso que el tiempo de los hombres se ajuste al aparato de producción, que este pueda utilizar el tiempo de vida, el tiempo de existencia de los hombres" (Foucault, 1991: 130).

De Coster (1996) sostiene que, en el capitalismo actual, la dilución de la frontera entre tiempo de trabajo y de no trabajo se debe a la búsqueda de optimización de las condiciones de uso del factor trabajo. El trabajo invade al tiempo libre y (con menor frecuencia) viceversa. La razón de esta invasión yace en que es necesario sumar a las horas de trabajo los tiempos de información y de formación necesarios para las funciones ejercidas y los tiempos que deben estar disponibles, los cuales reducen la autonomía temporal del empleado ${ }^{6}$.

Siguiendo la tesis de la evolución del disciplinamiento del tiempo de Thompson (1967), la medición del tiempo de trabajo, encierra una relación simple: los empleados experimentan una distinción entre el tiempo perteneciente a sus empleadores y su "propio" tiempo. El empleador debe usar el tiempo de su trabajo $y$ procurar que este no sea derrochado: no la tarea sino el valor del tiempo es lo dominante, cuando se reduce a dinero (Thompson, 1967:

6 A diferencia del modelo temporal de la sociedad industrial, en la sociedad red, "los tiempos de trabajo se transforman en su "naturaleza" (condiciones de trabajo, tecnología, gestión del personal...), en su administración (horarios...), sus ritmos (neotaylorización), su duración (reducción del tiempo de trabajo, jubilación avanzada, desempleo). Además, se precariza (debido a una multiplicidad de estatus, y a la segmentación...) y se diversifica (tercer sector, economía subterránea, trabajo en negro, economía doméstica...). El paradigma del empleo definido por el trabajo asalariado, único y estable, con una perspectiva de carrera continua $y$ progresiva y un vínculo salarial cerrado se modifica; poniéndose al servicio de la optimización temporal de la movilización de factores productivos, la extensión de la duración de funcionamiento de los equipamientos, $y$ la producción de riqueza concentrada aunque producida a partir de la deslocalización (Gaullier, citado por Luongo, 2005). 
61). El autor se preocupa por el sentido del tiempo condicionado por la tecnología y por la medición del tiempo como medio de explotación en el trabajo.

Lo que Thompson analiza no es el proceso de disciplinamiento del tiempo en la transición hacia el "industrialismo", sino hacia el capitalismo industrial, el cual posee sus propias características definidas. En esta transición, las luchas se definen principalmente por luchas alrededor del tiempo: finalmente los trabajadores aprenden la lección y comienzan a luchar no contra el reloj sino por alcanzar mayores niveles de autonomía temporal (lucha por menos horas de trabajo, por pago de horas extras, etc.): estos han aprendido que "el tiempo es dinero" (Thompson, 1967: 86). Este proceso se asemeja a una internalización o interiorización de la disciplina, pero el autor se pregunta hasta qué punto fue impuesta $y$ hasta cuánto fue asumida voluntariamente por los trabajadores. En las sociedades capitalistas maduras, todo tiempo debe ser consumido, comercializado, utilizado: "pasar el tiempo" constituye una ofensa para la fuerza laboral. La disciplina fue finalmente internalizada (Thompson, 1967: 91).

La gran narrativa de la Modernidad suponía la inserción de los agentes en la historia como poseedores de un pasado definitivo y un futuro predecible (Giddens, 1991: 12). La perspectiva de largo plazo suponía logros acumulativos. Esta gran narrativa de la historia, se disuelve una vez que se produce una pérdida de la creencia en el progreso. En el nuevo capitalismo, la experiencia personal se encuentra a la deriva: "La consigna nada a largo plazo desorienta la acción planificada, disuelve los vínculos de confianza y compromiso y separa la voluntad del comportamiento" (Sennet, 1998: 31). El tiempo histórico es expropiado de la vida de los sujetos, imponiéndose prácticas ligadas sólo al presente. Se deben elegir estrategias día a día.

El desarrollo del tiempo libre ha acentuado la oposición tiempo de trabajo-tiempo de no trabajo, pero como el tiempo de trabajo es el vector que contamina al resto del tiempo, la rentabilización del tiempo se ha acentuado (Boisard, 1984). En el mismo sentido, Bessin (1998) sostiene que en el modelo capitalista actual, la lentitud se encuentra estigmatizada, ya que nos encontramos en un paradigma epistemológico dentro del cual la racionalidad económica reposa sobre la rentabilidad temporal $y$ en el cual las innovaciones tecnológicas se derraman en la sociedad por medio de la velocidad de los modelos difusionistas del marketing. El tiempo es dinero en el sentido de que su inversión debe generar un retorno en metálico.

Jurczyk (1991) formula la tesis según la cual la vida cotidiana toma el carácter del trabajo, lo que traería como consecuencia un abuso temporal, fenómeno cada vez más expandido en las sociedades occidentales. Los individuos se ven obligados a reorganizar sus vidas día a día, o semana a semana. Esto ocurre con mayor acento en las empresas transnacionales. Cuanto mayor es la flexibilidad e incertidumbre exterior, agrega Jurczyk, mayor es la tendencia hacia la búsqueda de estabilidad y flexibilidad interior. La estabilidad interna va acompañada de una capacidad de actuar de manera flexible. De esta manera, el sujeto se repliega más hacia sí mismo, buscando la salvación individual? ${ }^{7}$.

Schehr (1999) prefiere hablar de una temporalidad social dominante, en la cual la temporalidad laboral juega un papel preponderante. El horizonte temporal de nuestras sociedades parece más que nunca caracterizarse por la incertidumbre y lo efímero sobre un fondo de crisis de legitimidad de la temporalidad social dominante. El autor presenta el ejemplo de los jóvenes fuertemente influidos por una ética de trabajo en la cual el presente queda suspendido —en detrimento de sus posibilidades - a una inserción definitiva aunque indeterminada en el mercado de trabajo: se podría hablar de una heterodeterminación cultural del tiempo en función de un exterior no manejable.

La gestión de la carrera de los managers, tan bien analizada por Luci (2007), se presenta en términos de una extrema movilidad, la cual genera una hipervalorización del tiempo presente: si en un momento, el diploma aseguraba oportunidades de promoción dentro de la

Laidi acuña el concepto de presente autárquico, en el cual este se desaferra del pasado y del futuro y cuyos ejes pasan a ser la autorreferencia y la autosuficiencia (Laidi, citado por Luongo, 2005). 
empresa, las cuales llevaban al desarrollo de una carrera, al hacerse evidente un alto porcentaje de desempleo entre este grupo social, si bien diferente en magnitud respecto a los sectores menos favorecidos, comenzó a mostrar una realidad diferente a la de los últimos años: si bien las empresas continúan ofreciendo perspectivas de promoción a los elementos considerados provistos de mayor talento, la seguridad a largo plazo no está garantizada (Boltanski y Chiapello, 2002: 27).

Este sobredimensionamiento del tiempo presente se produce a través de un aceleramiento del tiempo, se debilita la capacidad de insertar un momento dado en una perspectiva histórica y se desvanece tanto el pasado como el futuro, emergiendo un presente omnipotente (Lechner, citado por Luongo, 2005). Este presente omnipotente se asemeja al de la sociedad informacional descrita por Castells (1998), la que se caracteriza por ser una cultura on-line, instantánea, atemporal y global. La contrapartida se presenta como la cultura de la urgencia, una cultura del fin inmediato de la vida, sin ninguna proyección de largo plazo, los proyectos mutan de forma desenfrenada: "Las culturas locales de urgencia son la expresión invertida de la atemporalidad global" (Castells, 1998: 87). Prima lo contingente, el sentido de la vida es la vivencia inmediatamente perceptible.

Dubar (2002) sostiene que al estar la organización centrada en la eficacia inmediata, buscando la flexibilidad máxima para hacer frente a la incertidumbre, el empleado que entra a trabajar en la empresa busca estabilizarse y adquirir competencias profesionales. Así, pueden suscitarse malentendidos, ya que si el cuadro ha depositado todo en su desarrollo profesional dentro de la empresa, la ruptura puede llegar a ser dramática. La carrera deviene aleatoria e incierta. Si esta tensión entre el cuadro y la empresa es difícil de resolver, ¿Cómo es posible que los managers internalicen la disciplina de la empresa, aún incluso cuando pueden llegar a mantener, de forma paralela, visiones instrumentales acerca de su trabajo en la organización?

Es necesario aclarar que en los ámbitos empresariales, actualmente se hace hincapié en valorar el tiempo fuera del trabajo de los cuadros, tiempo dedicado a la afectividad. Este tiempo involucra a las empresas debido a que la afectividad es un rasgo fundamental de la empresa red (Boltanski y Chiapello, 2002). El tiempo libre es tiempo en el cual es posible desplegar libremente la creatividad para desarrollar redes. La literatura de gestión empresarial se rebela contra la separación entre tiempo de trabajo $y$ fuera de trabajo, ya que esta división separa aspectos indisociables de la vida (la afectividad familiar $y$ de las redes) $y$ va en contra de la flexibilidad requerida en el trabajo en red.

Cada manager debe comportarse como si fuera un empresario (o en otros términos, un inversor: invirtiendo en redes, en aprendizaje, en la carrera $y$ también en amistades e incluso en las relaciones más íntimas), corriendo con los riesgos, la autonomía, la carga de trabajo y la escisión de la frontera de los tiempos de trabajo $y$ de no trabajo que esto implica indefectiblemente. El cuadro debe comportarse como capitalista, llevando consigo mismo el capital llamado "humano"8: lo humano pasa a constituir un conjunto de habilidades, destrezas y aptitudes propias de los hombres que adquieren valor de mercado. El nuevo emprendedor interioriza la contraposición capital/trabajo (López Ruiz, 2004). A diferencia de lo que sostiene el autor, la realidad muestra que el nuevo emprendedor diluye, borra y solapa más que interioriza dicha contraposición.

8 López Ruiz (2004) presenta dos momentos significativos del espíritu capitalista: por un lado, los primeros años del siglo XX, en la cual la figura arquetípica del emprendedor adquiere significancia, $y$ por otro, a partir de los años 50 , durante los cuales se produce una crisis de dicha figura y de la ética del trabajo protestante, pasando a prevalecer la ética del trabajo empresarial —el trabajo pasa a constituir un emprendimiento individual- $\mathrm{El}$ autor utiliza la teoría del capital humano, ideada por economistas de la Universidad de Chicago en los años 50, para sustentar su tesis. Este sostiene que dicha teoría fue adoptada por las doctrinas de la gestión empresarial en las décadas de los 90 y 2000 , las cuales definen al capital humano como un conjunto de capacidades, habilidades y destrezas de los seres humanos que deben ser valorizados $y$ gestionados. 


\section{DISPOSITIVOS DISCIPLINARIOS Y DE CONTROL}

Si la acumulación se produce en un contexto de determinadas relaciones sociales, entonces está directamente relacionada con la disciplina. Para que exista un cierto patrón de acumulación, además de un disciplinamiento de la clase trabajadora, es necesario que los mandos medios se encuadren dentro de determinada relación social ${ }^{9}$.

El objetivo de la disciplina es reproducir un orden (en este caso un orden productivo) y una estructura jerárquica. Productividad y disciplina no pueden estar planteadas de forma separada. Así pues, las técnicas de gestión de la fuerza laboral (de gestión del personal y de los recursos humanos en la jerga empresarial), si bien se muestran bajo el manto de la inevitabilidad de cambios globales, por lo que se hace necesario adaptarse a reglas de juego impuestas desde afuera, lo que queda oculto es la gestión del poder y la naturaleza política de la técnica ${ }^{10}$.

Tal como sostiene Palomino (2000), las nuevas formas de organización del trabajo inducen a una sujeción no ya directa sino indirecta de la fuerza de trabajo, en las cuales la relación laboral está mediada por las reglas del mercado más

$9 \quad$ “(...) el estudio de la disciplina y de forma articulada, la del control, permite evidenciar las complejas relaciones sociales que constituyen y conforman el proceso de trabajo como instancia de valorización; $y$ de tal manera explican los específicos supuestos que subyacen al diseño de su estructura y funcionamiento. La disciplina comprende una estrategia reguladora de su configuración y operatividad, estrategia múltiple y compleja dirigida a conseguir la adecuación productiva del comportamiento de los trabajadores" (Gaudemar, citado por Montes Cató, 2004: 53).

De este modo, las formas de producción y disciplina se articulan gestionando un adecuado equilibrio entre la materialidad del orden y la eficacia social de sus representaciones simbólicas, a fin de adecuar la tecnicidad de los medios de producción, las funciones de los trabajadores y las combinaciones sociales del trabajo a las necesidades del capital expresadas en una cuádruple exigencia: permitir la continuidad del poder, obtener los máximos resultados productivos, facilitar la minoración de los costes y lograr un control efectivo del trabajo (Gaudemar, citado por Montes Cató, 2004: 54). que por la misma organización. Cada empleado se transforma en inversor, que comparte el riesgo del capital puesto en juego.

Boltanski y Chiapello sostienen que la historia de la gestión empresarial es una historia de la sofisticación permanente de los medios de dominación de cuanto ocurre en la empresa y en su entorno. El control incluye a los mercados, la competencia, los clientes, la cadena de distribución, el comportamiento de los consumidores, la prensa, los poderes políticos y hasta a los mismos directores de las grandes empresas. Por lo tanto, una pregunta central que debe formularse en una investigación empírica acerca de la cuestión del control en los cuadros es ¿Cómo funciona el proceso de internalización de la disciplina?, ¿Cuáles son las nuevas modalidades de control de la nueva gestión empresarial? Si la exigencia hacia los cuadros es la del autocontrol ¿De qué manera se manifiesta dicho control?11

Como hemos visto hasta ahora, el objetivo de las empresas no es que sus empleados acaten los lineamientos de su dirección por medio de la coacción física, sino que es necesario movilizar a los cuadros con el objetivo de que estos interioricen ciertos valores y creencias y que logren hacer propios los objetivos primarios de las empresas. La interiorización debe ser "dulcificada" como para que parezca lo más voluntaria posible. El objetivo del involucramiento es la fidelización para con la misión de la empresa, o en otras palabras, extraer plusvalor con la menor dificultad posible.

Una clara caracterización que hace tanto la literatura sobre managament como las grandes empresas transnacionales acerca de las nuevas formas de gestión es la aparente eliminación o reducción al mínimo de las jerarquías. Esta oda a la horizontalidad diferencia al discurso de los cuadros desde los años 90 frente

Para controlar lo incontrolable (el mercado, los clientes, los cuadros), la única solución es que las personas se autocontrolen, desplazando la coacción de la exterioridad de los dispositivos organizacionales a la interioridad de las personas, concediendo capital importancia a la implicación del personal, relacionado con la motivación y movilización del personal y con el placer de trabajar (Boltanski y Chiapello, 2002: 127). 
a lo que anteriormente se caracterizaba como una gestión verticalista $y$ burocrática. La horizontalidad logra eliminar (al menos en el plano simbólico) el carácter conflictual de la relación laboral, para reemplazarlo por una relación armoniosa en el espacio de trabajo ${ }^{12}$.

El discurso de la horizontalidad y la democracia en la gestión, tiene como contracara la evaluación del rendimiento de forma individual, tomando en cuenta las opiniones de jefes y pares. Ahora la presión, la jerarquía $y$ el control provienen del exterior: "El" cliente y "El" mercado. De esta manera, el mercado decidirá si las decisiones de un cuadro son evaluadas positiva o negativamente al final de un período, por lo que es el mercado quien decide sobre la continuidad o no del cuadro en la empresa. Así, las decisiones que están tomando algunas empresas en cuanto a la reducción de la semana laboral de sus cuadros (no así de los obreros) está estrechamente relacionado con el llamado a la eliminación de la autoridad por parte de las empresas y la exigencia de estas de que sus cuadros deben autoorganizarse ${ }^{13} y$ autodisciplinarse, tomando en cuenta las exigencias del cliente $y$ del mercado.

En los eventos empresarios organizados fuera del horario de trabajo, dedicados principalmente a la recreación, todos participan de las diferentes actividades en igualdad de condiciones, consumen los mismos alimentos, se tratan de igual a igual. El objetivo es constituir a la empresa como punto de referencia en la constitución de grupos y relaciones sociales. Se hace sumo hincapié (especialmente en estos eventos) en la constitución de un nosotros frente a un otro (competidor), el cual constituye la amenaza. El objetivo de la alta dirigencia es que los cuadros se sientan orgullosos de trabajar en la empresa.

13 El trabajo del capitalismo conexionista, caracterizado por Boltanski y Chiapello (2002), implica el trabajo en equipos pequeños pluridisciplinarios, flexibles y autónomos. El equipo es el lugar de la autoorganización y el autocontrol. La dirección o, mejor dicho, la coordinación, orientación y evaluación del rendimiento de estos equipos es realizada por unos pocos, llamados líderes con visión. Estos líderes visionarios logran que el compromiso, fidelidad y adhesión de los cuadros a las empresas en las que trabajan, no necesite de la fuerza. Por otro lado, éstos otorgan sentido al trabajo.
CONCLUSIONES PRELIMINARES (INCLUYENDO

INTERROGANTES QUE ENMARQUEN UNA ESTRATEGIA DE INVESTIGACIÓN EMPÍRICA)

Con el objetivo de sensibilizar a la audiencia a la cual se dirige, el discurso de gestión empresarial manifestado de la forma más clara en los textos de management, debe ser capaz de brindar herramientas morales para desenvolverse en la vida cotidiana. Este tipo de discurso dirigido a los cuadros, demuestra la imperiosa necesidad de que estos adhieran a la buena marcha de las empresas donde trabajan y se comprometan a la obtención de beneficios (ganancias) de las mismas. Esta necesidad se debe a que, al estar menos sometidos a la necesidad de las clases obreras, la posibilidad de contraponerse al régimen capitalista aumenta (incluso por medio de una resistencia pasiva o un comportamiento indiferente en relación con los objetivos empresarios).

Los cuadros, especialmente los más jóvenes, necesitan encontrar motivos personales para el compromiso, por lo que las oportunidades que se les presentan adquieren el carácter de excitantes, plenas de pasión, portadoras de posibilidades de autonomía y autorrealización. Sin embargo, paralelamente con los anhelos de libertad, el capitalismo (o en su defecto, la empresa) debe proporcionar seguridad para los cuadros, demanda que en la actualidad es más difícil de satisfacer ${ }^{14}$.

Si tal como sostienen Boltanski y Chiapello (2002), en tanto que la justificación compagina simultáneamente las justificaciones individuales (motivos personales para adherirse al capitalismo) como las generales (en las cuales el compromiso para con el capitalismo sirve al

Boltanski y Chiapello (2002) establecen tres interrogantes a los cuales el capitalismo debe proporcionar una respuesta adecuada e históricamente situada a sus cuadros, de las cuales nos interesa plantear una que se relaciona con la cuestión de la justificación: ¿De qué manera puede el compromiso con el proceso de acumulación capitalista ser una fuente de entusiasmo incluso para aquellos que no serán los primeros en aprovecharse de los beneficios realizados? ¿Cómo responden los cuadros a esta cuestión? Es lo que un futuro trabajo empírico, basado en el debate teórico planteado aquí, debiera indagar. 
bien común), entonces se allana el camino para presentar un interrogante que no podrá ser resuelta en este trabajo, debido a que requiere de un anclaje empírico: ¿Cómo manifiestan los cuadros las diferentes formas de justificación dentro de las mismas empresas? ${ }^{15}$. La literatura relacionada al management (gestión de organizaciones en general y de empresas en particular) provee a los cuadros de suficientes herramientas de justificación de la forma de obtención de beneficios y con el objeto de resistir a las críticas (por parte de los subordinados $y$ de contextos sociales ajenos a las empresas) a la hora de llevar a cabo las recomendaciones que hacen los textos (generalmente en forma de casos de estudio) para tal propósito.

\section{BIBLIOGRAFÍA}

Adorno, T. Consignas. Buenos Aires: Amorrortu, 1973.

Bessin, M. "Vitesse et lenteur dans la vie sociale: prolongements d'un séminaire". Temporalistes 38. 1998. [Revista digital] En: <http://www.sociologics. org/temporalistes $>$ [consultado 17 de septiembre 2009].

Boisard, P. "Réduction de la durée du travail et transformations du modèle temporel". Temporalistes 2. 1984. [Revista digital] En: <http://www.sociologics.org/ temporalistes $>$ [consultado $17 \mathrm{de}$ septiembre 2009].

Boisard, P. "L'aménagement du temps de travail entre tyrannie du marché et

15 "Estas justificaciones deben apoyarse en argumentos lo suficientemente robustos como para ser aceptados como evidentes por un número lo suficientemente grande de gente, de manera que pueda contenerse o superarse la desesperanza o el nihilismo que el orden capitalista no deja de inspirar igualmente, no sólo entre quienes oprime, sino también, a veces, entre quienes tienen la tarea de mantenerlo $y$, a través de la educación, transmitir sus valores" (Boltanski y Chiapello, 2002: 46). choix individuels". Temporalistes 25 . 1993. [Revista digital] En: <http:// www.sociologics.org/temporalistes $>$ [consultado 17 de septiembre 2009].

Boltanski, L. y Chiapello, E. El nuevo espíritu del capitalismo. Madrid: Akal, 2002.

Bouffartigue, P. (dir.) et ál. Cadres: la grande ruptura. París: La Découverte, 2001.

Bouffartigue, P. Les cadres. Fin d'une figure sociale. París: La Dispute, 2001.

Brunel. Les managers de l'âme. Le développement personnel en entreprise, nouvelle forme de pouvoir? París: La Découverte, Coll. Entreprise \& société, 2004.

Castells, M. La era de la información. Vol. I: El poder de la identidad. Madrid: Alianza, 1998.

De Coster, M. "Les nouvelles technologies et l'évolution des temps de travail". Temporalistes 33. 1996. [Revista digital] En: <http://www.sociologics. org/temporalistes $>$ [consultado $17 \mathrm{de}$ septiembre 2009].

De Gauléjac. La société malade de la gestion: Idéologie gestionnaire, pouvoir managérial et harcélement social. París: Seuil, 2005.

Deleuze, G. Conversaciones 1972-1990. Valencia: Ed. Pre-textos, 1995.

Delfino, A. Tiempo y cotidianeidad en el mundo del no trabajo. Algunas hipótesis iniciales. 2004. (s/d).

Dubar, C. "Larticulation des temporalités dans la construction des identités personnelles: questions de recherche et problèmes d'interprétation". Temporalistes 44. 2002. [Revista digital] En: <http:// www.sociologics.org/temporalistes $>$ [consultado 17 de septiembre 2009]. 
Durand, J. P. y Le Floch, M. Ch. (dir.). La question du consentement au travail: De la servitude volontaire à l'implication contrainte. París: L'harmattan, 2006.

Flament, N. Une anthropologie des managers. París: PUF, Coll. Sciences socials, 2002.

Foucault, M. La verdad y las formas jurídicas. Barcelona: Gedisa, 1991.

Gadéa, Ch. Les cadres en France. Une énigme sociologique. París: Belin, Coll. Perspectives sociologiques, 2003.

Gaudemar, J.P. El orden y la producción. Nacimiento y formas de la disciplina de fábrica. España: Ed. Trotta, 1991.

Giddens, A. As conseqüências da modernidade. São Paulo: Ed. UNESP, 1991.

Jurczyk, K. "L'impact de l'organisation de la vie quotidienne sur la flexibilite du travail en Alemagne". Temporalistes 17. 1991. [Revista digital] En: <http:// www.sociologics.org/temporalistes $>$ [consultado 17 de septiembre 2009].

Lechner, N. Las sombras del mañana. La dimensión subjetiva de la política. Santiago: Lom ediciones, 2002.

Livian, Y. F. (comp.). Etre cadre, quel travail? París: Anact, 2006.

López Ruiz, O. "O "ethos" dos executivos das transnacionais e o espírito do capitalismo". [Tesis de doctorado defendida en la Universidad estadual de Campinas, SP]. 2004.

Luci, F. "Managers de empresas multinacionales: La gestión de la carrera en las grandes firmas". Ponencia presentada en el VIII Congreso Nacional de Estudios del Trabajo. Facultad de Ciencias Económicas, Universidad de Buenos Aires. 2007.
Luongo, M.E. "Un tiempo incierto. La socialización en el trabajo en un contexto de transformaciones". Ponencia presentada en el $7 \mathrm{mo}$. Congreso de ASET. Buenos Aires. 2005.

Montes, J. "Subordinación y dominación en los espacios de trabajo. Estudio sobre la disciplina y sus formas de expresión". Athenea Digital 8. 2005. [Revista digital] En: <http://antalya.uab.es/athenea/ num $8 /$ montes.pdf $>$ [consultado 17 de septiembre 2009].

Palomino, H. "Trabajo y teoría social: conceptos clásicos y tendencias contemporáneas. Del trabajo asalariado a la sujeción indirecta del trabajo al capital. Un ensayo sobre los cambios contemporáneos en las relaciones sociales". Ponencia presentada en el III Congreso Latinoamericano de Sociología del Trabajo. Buenos Aires. 2000 .

Pronovost, G. "Manquons-nous de temps ?". Temporalistes 37. 1998. [Revista digital]. En: <http://www.sociologics. org/temporalistes $>$ [consultado 17 de septiembre 2009].

Schehr, S. (1999). "La conquête de l'autonomie temporelle". Temporalistes 40. [Revista digital] En: <http://www.sociologics. org/temporalistes $>$ [consultado 17 de septiembre 2009].

Sennet, R. La corrosión del carácter. Barcelona: Anagrama, 1998.

Sgrazzutti, J. La organización del tiempo libre en dictaduras europeas $y$ en Argentina entre 1922 y 1955. 2004. [Artículo digital] En: <http://seneca.uab.es/hmic> [consultado 17 de septiembre 2009].

Thompson, E. P. "Time, work discipline and industrial capitalism". Past and present 38. Oxford. 1967. 